\title{
Role of downregulated ADARB1 in lung squamous cell carcinoma
}

\author{
XIANG WANG ${ }^{1,2}$, XINXIN REN $^{3}$, WANLI LIU ${ }^{1,2}$, XI CHEN $^{1,2}$, JIE WEI $^{1,2}$, \\ ZHICHENG GONG ${ }^{1,2}$, YUANLIANG YAN ${ }^{1,2}$ and ZHIJIE XU ${ }^{4}$ \\ ${ }^{1}$ Department of Pharmacy; ${ }^{2}$ National Clinical Research Center for Geriatric Disorders, Xiangya Hospital, \\ Central South University; ${ }^{3}$ Center for Molecular Medicine, Xiangya Hospital, Key Laboratory of \\ Molecular Radiation Oncology of Hunan Province, Central South University; ${ }^{4}$ Department of Pathology, \\ Xiangya Hospital, Central South University, Changsha, Hunan 410008, P.R. China
}

Received July 10, 2019; Accepted December 17, 2019

DOI: $10.3892 / \mathrm{mmr} .2020 .10958$

\begin{abstract}
Non-small cell lung cancer (NSCLC) is prevalent worldwide. Lung squamous cell carcinoma (LUSC) is one of the main subtypes of NSCLC yet, currently, few biomarkers are available for the diagnosis of LUSC. The present study aimed to investigate the expression and role of adenosine deaminase RNA specific B1 (ADARB1) in lung squamous cell carcinoma (LUSC). Integrative bioinformatics analysis was used to identify the effects of ADARB1 expression on the occurrence and prognosis of LUSC. The expression of ADARB1 was further examined by immunohistochemistry (IHC). Bioinformatics analysis suggested that ADARB1 was downregulated in LUSC, serving as a potential tumor suppressor, and these results were verified by IHC performed on a lung cancer tissue array. Clinical studies suggested that ADARB1 expression and methylation levels were significantly associated with patient characteristics in LUSC. Moreover, ADARB1 global methylation levels were upregulated in LUSC tissues compared with normal lung tissues. Higher methylation levels of cg24063645 were associated with shorter overall survival time of patients with LUSC. A negative correlation was identified between ADARB1 and epidermal growth factor receptor (EGFR) expression in LUSC. Using the Gene Expression Omnibus database, it was suggested that the expression of ADARB1 in LUSC was significantly different compared with that in lung adenocarcinoma. Furthermore, protein-protein interactions were studied and a biological process annotation analysis was conducted. The present
\end{abstract}

Correspondence to: Dr Yuanliang Yan, Department of Pharmacy, Xiangya Hospital, Central South University, 87 Xiangya Road, Changsha, Hunan 410008, P.R. China

E-mail: yanyuanliang@csu.edu.cn

Dr Zhijie Xu, Department of Pathology, Xiangya Hospital, Central South University, 87 Xiangya Road, Changsha, Hunan 410008, P.R. China

E-mail: xzj1322007@csu.edu.cn

Key words: adenosine deaminase RNA specific B1, expression, methylation, survival, therapeutics study suggested that ADARB1 was downregulated in LUSC; therefore, ADARB1 may serve as a specific biomarker and a potential therapeutic target for LUSC.

\section{Introduction}

Lung cancer was the leading cause of cancer-related death worldwide in 2015 and non-small cell lung cancer (NSCLC) accounts for $\sim 85 \%$ of lung cancer cases (1). Lung squamous cell carcinoma (LUSC) is one of the main subtypes of NSCLC. A previous study indicated that the 5-year overall survival (OS) rate for patients with LUSC is $17.7 \%$ due to late diagnosis (2). In addition, there have been limited opinions for LUSC treatment compared to lung adenocarcinoma (LUAD) although progress has been made in the treatment of NSCLC (3-5). The reason is partly because there are more molecular abnormalities for LUSC in the discoidin domain receptor tyrosine kinase 2 and fibroblast growth factor receptor 1 genes (6). Early identification of the disease would reduce the mortality rate of patients with LUSC who could benefit most from effective therapies (7). However, few biomarkers are currently available for the effective diagnosis of LUSC, which would enable patients to receive timely treatment (8). Thus, identifying novel target molecules is essential for improving the diagnosis and treatment of LUSC.

Adenosine deaminase RNA specific B1 (ADARB1) is a member of the adenosine deaminases acting on RNA (ADAR) family, which exhibit enzymatic editing activity in a process known as adenosine-to-inosine (A-to-I) editing (9). Recently, research has indicated an association between ADARB1 and cancer. A previous study reported that ADARB1, an indispensable marker in brain development and function, could inhibit the proliferation and progression of glioblastoma (10). In terms of esophageal squamous cell carcinoma, Chen et al (11) reported that ADARB1 induces apoptosis and suppresses tumor growth by editing insulin-like growth factor binding protein 7 mRNA. Additionally, ADARB1 can regulate exon 12A, an exon from the heterogeneous nuclear ribonucleoprotein $\mathrm{L}$ like transcript, to promote cancer cell survival (12). However, the relationship between ADARB1 and LUSC has not been investigated.

Therefore, the present study aimed to investigate the role of ADARB1 in LUSC. The results suggested that ADARB1 was downregulated in LUSC tissues and cell lines. The 
expression and methylation status of ADARB1 were significantly associated with clinical characteristics and prognosis. Additionally, the GSE10245 dataset (13), acquired from the Gene Expression Omnibus (GEO) (14) database, suggested that ADARB1 expression levels in LUSC were lower than in LUAD. Finally, functional enrichment analysis of ADARB1-associated co-expression genes was conducted and a protein-protein interaction (PPI) network was established. The results of the Gene Ontology (GO) analysis suggested that the main biological processes ('metabolic process' and 'biological regulation'), cellular components ('nucleus') and molecular functions ('protein binding') were associated with ADARB1. In addition, ADARB1 expression was significantly related to the Kyoto Encyclopedia of Genes and Genomes (KEGG) terms 'ubiquitin mediated proteolysis' and 'Wnt signaling pathway'.

\section{Materials and methods}

Data acquisition and reanalysis using bioinformatics methods. Several bioinformatics web resources were used to reanalyze data concerning ADARB1 (Table SI). The Genome Mining (GE-mini) database was employed to examine gene expression profiles over a number of tissue types, including tumors (15). The Cancer Cell Line Encyclopedia (CCLE) project is a public database containing detailed genetic and pharmacological characterization of numerous cell lines (16). The GE-mini and CCLE databases were used to identify the expression profiles of ADARB1 in LUSC tissues and cell lines. Oncomine (17), University of Alabama Cancer Database (UALCAN) (18) and Gene Expression Profiling Interactive Analysis (GEPIA) (19) were used to verify the results. The GEPIA database was also used to identify the Pearson correlation between ADARB1 and epidermal growth factor receptor (EGFR) in LUSC.

Wanderer is an interactive platform, which provides human cancer gene expression and DNA methylation data. This platform was used to evaluate the relationship between ADARB1 levels and patient clinical characteristics (20). DiseaseMeth (version 2.0) is a human disease methylation database, which was used to analyze ADARB1 global methylation levels in LUSC (21). The MethSurv tool was used to analyze the association between ADARB1 methylation and LUSC prognosis by using the mean value to dichotomize methylation profiles of patients (22). The GSE10245 dataset, downloaded from the GEO database, provided ADARB1 expression profiles in LUAD and LUSC samples.

A dataset LUSC (TCGA, Nature 2012) (23) from the cBioportal (24) web tool was used to obtain gene co-expression data for ADARB1 in LUSC. The Search Tool for the Retrieval of Interacting Genes/Proteins (STRING) database (25) and Cytoscape software (26) were then used to build a PPI network of the co-expression genes. Moreover, GO and KEGG (27) pathway analyses were conducted, using the Web-based Gene Set Analysis Toolkit (WebGestalt) (28) and the Database for Annotation, Visualization and Integrated Discovery (DAVID) (29) bioinformatics resources, respectively.

Immunohistochemistry (IHC) analysis of a lung cancer tissue array. The LUC481 lung cancer tissue array and information regarding the cancer subtypes of the array were obtained from
Fanpu Biotech, Inc. IHC was performed using the Histomouse SP Broad Spectrum DAB kit (Invitrogen; Thermo Fisher Scientific, Inc.) according to the manufacturer's protocol. Following antigen retrieval which was performed by microwaving in $10 \mathrm{mM}$ citrate buffer ( $\mathrm{pH}$ 6.0), paraffin-embedded sections were immunostained using a streptavidin peroxidase procedure. After blocking the samples with $3 \%$ bovine serum albumin (100 ml, SW3015, Beijing Solarbio Science $\&$ Technology Co., Ltd.) for $15 \mathrm{~min}$ at room temperature, the primary antibody against ADARB1 (cat. no. sc-73409, Santa Cruz Biotechnology, Inc., 1:100 dilution) was added and samples were incubated at $4^{\circ} \mathrm{C}$ overnight. Following washing with PBS, the horseradish peroxidase (HRP)-conjugated polymer second antibody (PV-6000, OriGene Technologies, Inc.) was added and samples were incubated for $30 \mathrm{~min}$ at room temperature. The signal was examined using a 3,3'-diaminobenzidine solution. Subsequently, four fields of view of the stained sections were independently observed in a light microscope at magnifications of $x 4$ and $x 20$ by two pathologists. The staining intensity of each protein was divided into four grades (intensity scores): 0 (negative), 1 (weak brown), 2 (moderate brown) and 3 (strong brown). The percentage of positive cells was scored as 0 ( $\leq 10 \%), 1$ (11-25\%), 2 (26-50\%), 3 (51-75\%) and $4(>75 \%)$ (30). The final staining score was calculated using the following formula: Intensity score $\mathrm{x}$ percentage score (1). A final score $>1$ was defined as high expression and final scores $\leq 1$ were defined as low expression.

Statistical analysis. Statistical analyses were performed using SPSS software (version 12.0; SPSS, Inc.). The data are presented as the mean $\pm \mathrm{SD}$. A paired-sample t-test was used for normally distributed and continuous variables were statistically analyzed by one-way analysis of variance with LSD post hoc test. Multivariate analysis was accomplished utilizing the Cox regression model to identify independence of accepted clinical parameters and the ordinal variables were tested with Kruskall-Wallis test followed by Dunn's post-hoc test. The Kaplan-Meier analysis was statistically tested by the log-rank method. $\mathrm{P}<0.05$ was considered to indicate a statistically significant difference.

\section{Results}

ADARB1 is downregulated in LUSC tissues and cell lines. By analyzing several databases, the transcriptional levels of ADARB1 in LUSC tissues and cell lines were compared with those observed in normal lung tissue and cells. GE-mini analysis revealed that ADARB1 mRNA expression was significantly decreased in LUSC tissues (Fig. 1A). The CCLE database suggested that ADARB1 mRNA expression was significantly downregulated in $\sim 10$ LUSC cell lines compared with immortalized lung epithelial cell lines $(\mathrm{P}=0.001$; Fig. 1B). To further investigate these observations, the Oncomine, UALCAN and GEPIA databases were employed. Analysis with all three databases suggested that ADARB1 was expressed at significantly lower levels in LUSC tissues compared with in normal lung tissues (Fig. 1C-E). Furthermore, IHC analysis, based on a lung cancer array containing 16 normal samples, 12 LUSC samples and 10 LUAD samples, suggested that ADARB1 


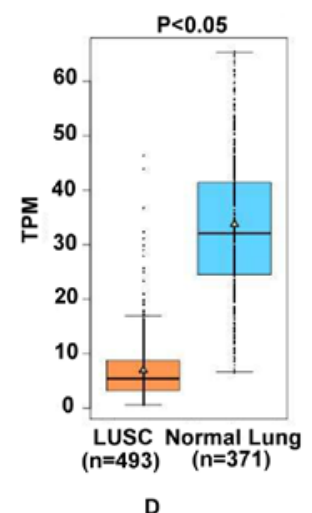

B

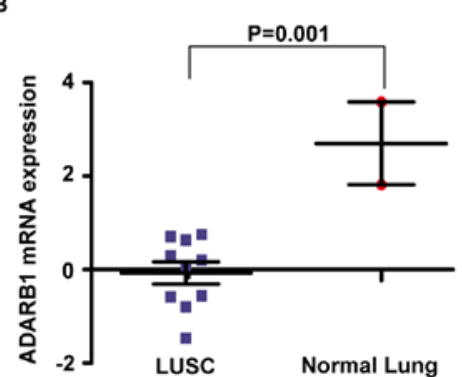

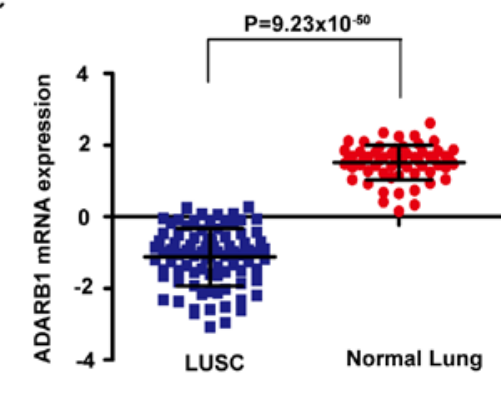

$\mathbf{E}$

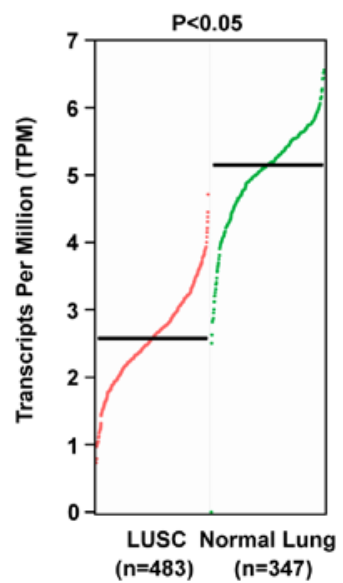

Figure 1. Downregulation of ADARB1 in LUSC tissues and cell lines, as indicated by several databases. The expression of ADARB1 was analyzed by the (A) Genome Mining, (B) Cancer Cell Line Encyclopedia, (C) Oncomine, (D) University of Alabama Cancer Database and (E) Gene Expression Profiling Interactive Analysis databases. ADARB1, adenosine deaminase RNA specific B1; LUSC, lung squamous cell carcinoma; TPM, transcripts per million.

was significantly downregulated in LUSC samples compared with normal lung samples $\left(\mathrm{P}=1.58 \times 10^{-8}\right.$; Fig. $2 \mathrm{~A}$ and $\left.\mathrm{B}\right)$. In summary, the downregulation of ADARB1 expression levels in LUSC tissues and cell lines suggested that ADARB1 may be a promising anti-oncogene in LUSC.

ADARBI could be used for the identification of LUSC and $L U A D$. The results of the IHC analysis further suggested that ADARB1 was downregulated in LUAD tissues compared with normal lung tissues, which was consistent with our previous study ( $\mathrm{P}=0.024$; Fig. 2A and B) (31). Furthermore, ADARB1 expression levels were significantly lower in LUSC tissues than in LUAD tissues ( $\mathrm{P}=0.025$; Fig. $2 \mathrm{~A}$ and $\mathrm{B}$ ). Subsequently, the GSE10245 dataset, obtained from GEO, suggested that ADARB1 was expressed at a lower level in LUSC compared with LUAD ( $\mathrm{P}=0.031$; Fig. $3 \mathrm{~A})$. Furthermore, this significant difference was verified by the CCLE database $(\mathrm{P}=0.008$; Fig. 3B). Therefore, these results suggested that ADARB1 expression profiles may serve as indicators for the clinical diagnosis of LUSC and LUAD.

Relationship between ADARB1 expression and the clinical characteristics of patients with LUSC. The effects of ADARB1 expression on the clinical characteristics of patients with LUSC were examined. Clinical data were downloaded from the Wanderer database, and the clinical characteristic parameters are summarized in Table I. The expression of ADARB1 was significantly associated with the pathologic $\mathrm{N}$ stage $(\mathrm{P}=0.045)$, pathologic $\mathrm{M}$ stage $(\mathrm{P}=0.012)$ and tobacco smoking history indicator $(\mathrm{P}=0.008)$. Furthermore, multivariate analysis of the clinical data suggested that the pathologic $\mathrm{N}$ stage $(\mathrm{P}=0.020)$, pathologic $\mathrm{M}$ stage $(\mathrm{P}=0.003)$ and tobacco smoking history indicator $(\mathrm{P}=0.017)$ were independently associated with ADARB1 expression in patients with LUSC (Table II).

ADARB1 methylation is related to the clinical characteristics of patients with LUSC. The ADARB1 global methylation levels in LUSC were evaluated using the DiseaseMeth database. ADARB1 displayed significantly higher levels of methylation in LUSC samples compared with in normal lung samples $\left(\mathrm{P}=1.62 \times 10^{-12}\right.$; Fig. $\left.4 \mathrm{~A}\right)$. The highest methylation value of $\operatorname{cg} 24063645$ in ADARB1 was identified using the Wanderer and MethSurv databases $\left(\mathrm{P}=3.60 \times 10^{-19}\right.$; Fig. 4B; Table SII). Subsequently, the association between cg24063645 methylation and the clinical characteristics of patients with LUSC was analyzed. The methylation of $\operatorname{cg} 24063645$ of ADARB1 was significantly associated with the Karnofsky score of patients with LUSC ( $\mathrm{P}=0.025$; Table III). Moreover, higher methylation levels of $\operatorname{cg} 24063645$ of ADARB1 were associated with shorter OS of patients with LUSC ( $\mathrm{P}=0.044$; Fig. 4C). Therefore, the results suggested that $A D A R B 1$ methylation levels may serve a role in the prognosis of patients with LUSC.

ADARB1 expression is negatively correlated with EGFR expression in LUSC tissues. EGFR is an oncogenic driver that contributes to the activation and development of lung cancer (7). Previous studies have indicated a poor correlation between EGFR expression and the overall response rate in lung cancer, particularly in $\operatorname{LUSC}(7,32,33)$. The GEPIA database was employed to evaluate the relationship between ADARB1 
A
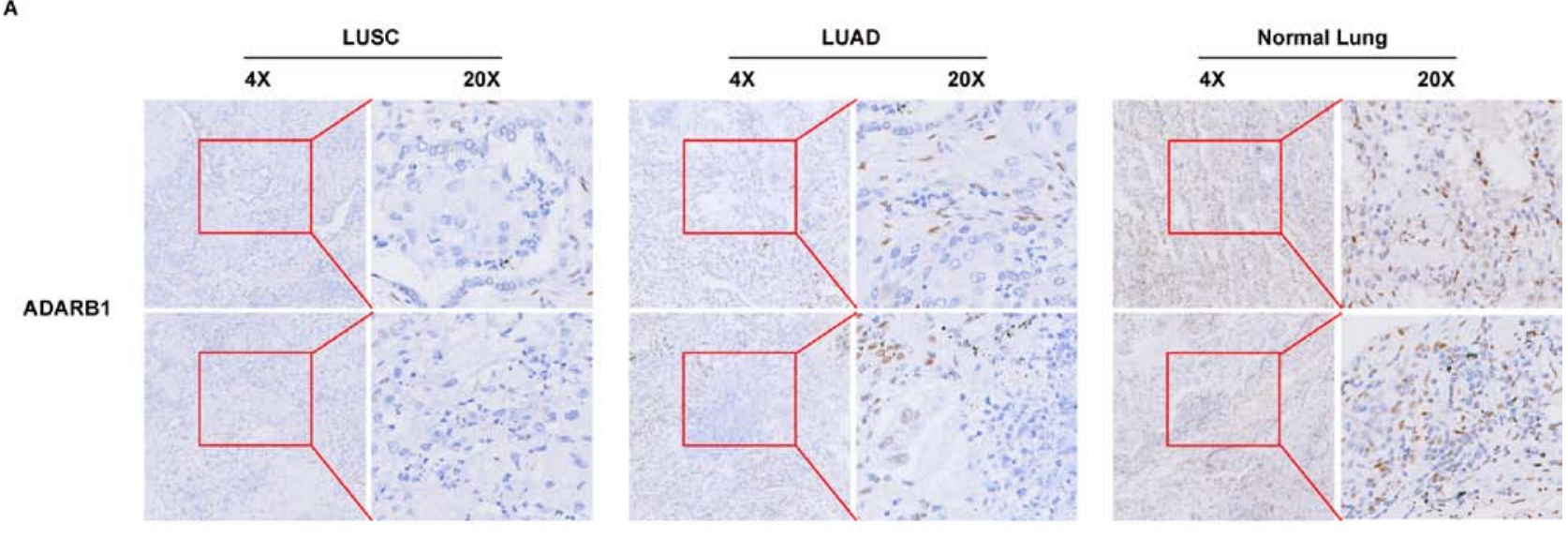

B

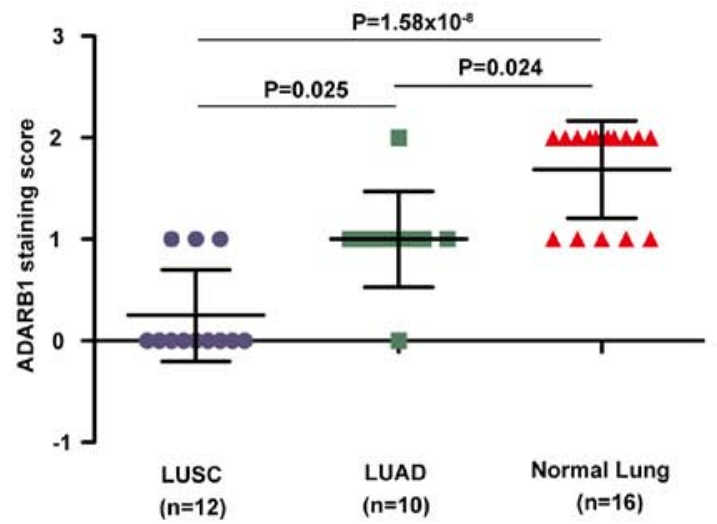

Figure 2. Downregulation of ADARB1 in the lung cancer tissue array. (A) Immunohistochemistry was used to examine the level of ADARB1 expression in a commercial lung cancer tissue array. (B) Protein levels of ADARB1 were significantly downregulated in the lung tissue of patients with LUSC or LUAD compared with normal lung tissue. ADARB1 expression was significantly decreased in LUSC tissues compared with LUAD tissue samples. ADARB1, adenosine deaminase RNA specific B1; LUAD, lung adenocarcinoma; LUSC, lung squamous cell carcinoma.
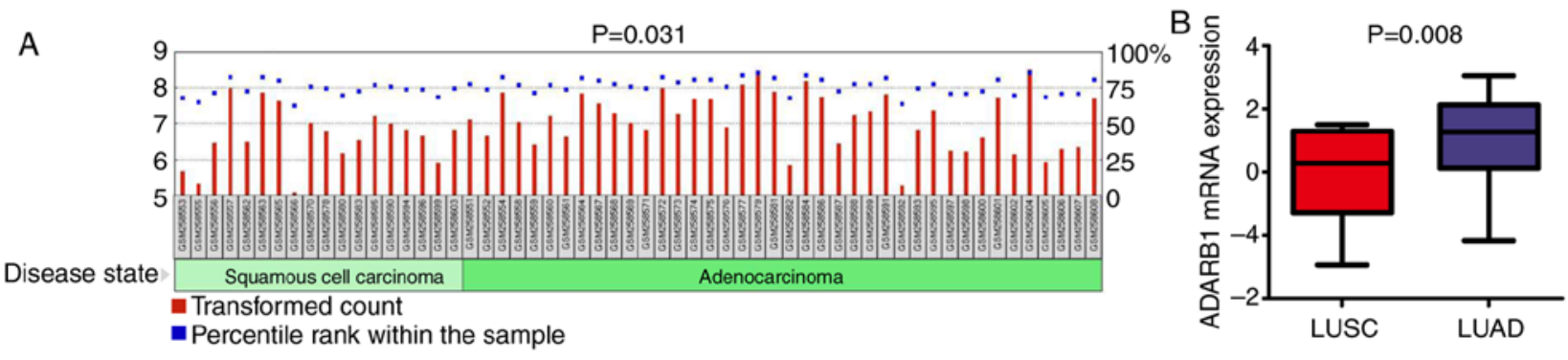

Figure 3. ADARB1 expression is significantly different between LUSC and LUAD. (A) Dataset GSE10245, obtained from Gene Expression Omnibus, suggested that ADARB1 was expressed at a lower level in LUSC compared with LUAD. (B) Difference in ADARB1 expression in LUSC and LUAD cell lines, as determined by Cancer Cell Line Encyclopedia analysis. ADARB1, adenosine deaminase RNA specific B1; LUAD, lung adenocarcinoma; LUSC, lung squamous cell carcinoma.

and EGFR. A negative correlation between ADARB1 and EGFR transcript levels in tissues of patients with LUSC was identified ( $\mathrm{P}=7.80 \times 10^{-6} ; \mathrm{R}=-0.16$; Fig. 5), which suggested that ADARB1 acted as an anti-cancer gene and might be linked to EGFR.

Network analysis of genes co-expressed with ADARB1. To further investigate the biological function of ADARB1, a functional enrichment analysis of its associated co-expressed genes was conducted. Firstly, 14,102 genes co-expressed with ADARB1 in LUSC were identified using the cBioPortal database, and a volcano plot was generated to display the association between groups with altered and unaltered ADARB1 expression (Fig. 6A). Based on the criteria of llog ratiol $>0.3$ and $\mathrm{P}<0.05,158$ ADARB1-associated, co-differentially expressed genes (co-DEGs) were identified (Table SIII). A protein-protein interaction network was generated using the STRING database and Cytoscape software (Fig. 6B). Moreover, WebGestalt was used to analyze GO terms (Fig. 6C) and indicated that the main biological processes of the co-DEGs were primarily enriched in 'metabolic process' and 'biological regulation'. Moreover, Gan et al (34) reported that ADARB1 participated 
Table I. Association between clinical characteristic parameters and the expression of adenosine deaminase RNA specific B1 in lung squamous cell carcinoma.

\begin{tabular}{|c|c|c|c|}
\hline Variable & $\mathrm{n}$ & Mean \pm SD & P-value \\
\hline Pathologic $\mathrm{T}$ & & & 0.433 \\
\hline $\mathrm{T} 1 / \mathrm{T} 1 \mathrm{a} / \mathrm{T} 1 \mathrm{~b}$ & 100 & $8.148 \pm 0.924$ & \\
\hline $\mathrm{T} 2 / \mathrm{T} 2 \mathrm{a} / \mathrm{T} 2 \mathrm{~b}$ & 252 & $8.103 \pm 1.035$ & \\
\hline $\mathrm{T} 3$ & 48 & $8.367 \pm 0.967$ & \\
\hline $\mathrm{T} 4$ & 19 & $8.155 \pm 1.232$ & \\
\hline Pathologic N & & & 0.045 \\
\hline N0 & 262 & $8.134 \pm 1.008$ & \\
\hline N1 & 111 & $8.236 \pm 0.955$ & \\
\hline $\mathrm{N} 2$ & 5 & $7.533 \pm 1.031$ & \\
\hline N3 & 6 & $9.050 \pm 0.584$ & \\
\hline Pathologic M & & & 0.012 \\
\hline M0 & 357 & $8.113 \pm 0.996$ & \\
\hline $\mathrm{M} 1 / \mathrm{M} 1 \mathrm{a}$ & 6 & $7.576 \pm 0.607$ & \\
\hline MX & 50 & $8.511 \pm 1.081$ & \\
\hline Pathologic tumor stage & & & 0.078 \\
\hline $\mathrm{I} / \mathrm{IA} / \mathrm{IB}$ & 211 & $8.116 \pm 0.985$ & \\
\hline IIA/IIB & 123 & $8.315 \pm 0.981$ & \\
\hline IIIA/IIIB & 76 & $8.013 \pm 1.122$ & \\
\hline IV & 6 & $7.576 \pm 0.607$ & \\
\hline Ethnicity & & & 0.133 \\
\hline White & 297 & $8.190 \pm 1.033$ & \\
\hline Black/African American & 16 & $8.208 \pm 0.809$ & \\
\hline Asian & 9 & $8.888 \pm 1.028$ & \\
\hline Tobacco smoking history indicator & & & 0.008 \\
\hline Current reformed smoker for $>15$ years & 71 & $8.404 \pm 0.991$ & \\
\hline Current reformed smoker for $\leq 15$ years & 211 & $8.018 \pm 1.007$ & \\
\hline Current reformed smoker, duration not specified & 1 & $9.922 \pm 0.000$ & \\
\hline Lifelong non-smoker & 17 & $8.533 \pm 1.042$ & \\
\hline Current smoker & 112 & $8.137 \pm 0.977$ & \\
\hline Age & & & 0.857 \\
\hline$\leq 60$ years & 89 & $8.133 \pm 1.093$ & \\
\hline$>60$ years & 322 & $8.155 \pm 0.994$ & \\
\hline Karnofsky score & & & 0.236 \\
\hline $0-70$ & 47 & $7.939 \pm 1.233$ & \\
\hline 80 & 14 & $7.910 \pm 0.884$ & \\
\hline 90 & 22 & $8.173 \pm 0.948$ & \\
\hline 100 & 10 & $8.687 \pm 0.957$ & \\
\hline Eastern Cooperative Oncology Group score & & & 0.079 \\
\hline 0 & 60 & $8.470 \pm 1.099$ & \\
\hline 1 & 71 & $8.208 \pm 0.978$ & \\
\hline 2 & 20 & $8.007 \pm 1.061$ & \\
\hline 3 & 3 & $7.211 \pm 0.597$ & \\
\hline
\end{tabular}

Values in bold are significant.

in the regulation of pancreatic islet and $\beta$-cell function. Furthermore, deficient or hyperactive ADARB1 expression is associated with a number of human diseases, including immunological disorders, amyotrophic lateral sclerosis, epilepsy and cancer (35). Therefore, it was speculated that ADARB1 might serve a role in LUSC via metabolic processes and biological regulation. In addition, for cellular components and molecular functions, the co-DEGs were primarily enriched in 'nucleus' 
Table II. Multivariate analysis of clinical data related to adenosine deaminase RNA specific B1 expression in lung squamous cell carcinoma.

\begin{tabular}{lccccc}
\hline Characteristic & $\begin{array}{c}\text { Type III } \\
\text { sum of squares }\end{array}$ & $\begin{array}{c}\text { Degrees of } \\
\text { freedom }\end{array}$ & Mean square & F-value & P-value \\
\hline Pathologic N & 11.32 & 4 & 2.83 & 2.96 & 0.020 \\
Pathologic M & 11.50 & 2 & 5.75 & 6.02 & 0.003 \\
Tobacco smoking history indicator & 9.88 & 3 & 3.30 & 3.45 & 0.017 \\
\hline
\end{tabular}

A

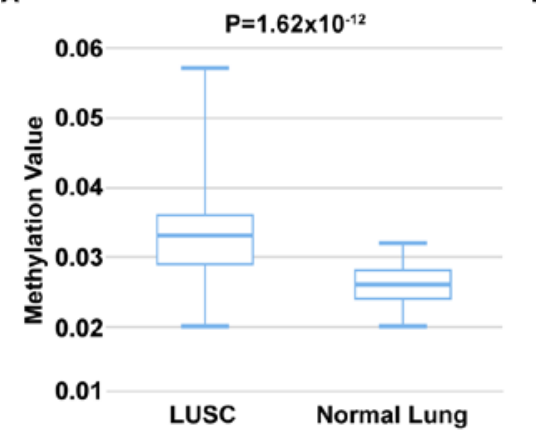

B

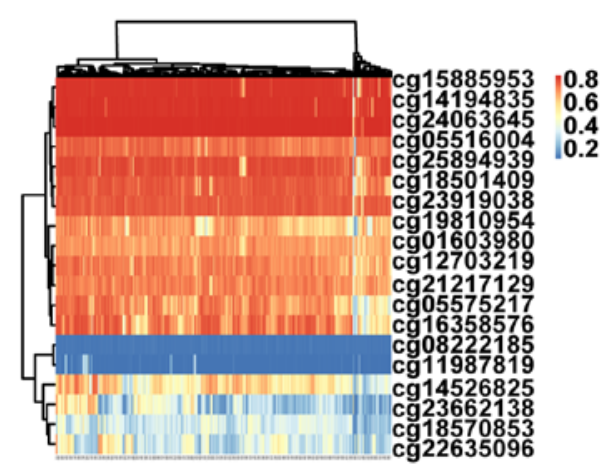

C

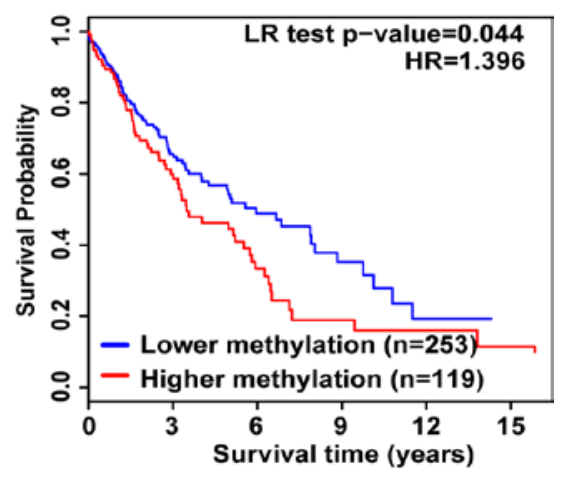

Figure 4. Methylation values of ADARB1 in patients with LUSC. (A) Global methylation of ADARB1 in LUSC samples compared with normal samples, using DiseaseMeth. (B) Heat map () acquired from MethSurv described the methylation sites of ADARB1 in LUSC. Red represents upregulation and blue represents downregulation. (C) Relationship between methylation values of cg24063645 of ADARB1 and overall survival time in LUSC. ADARB1, adenosine deaminase RNA specific B1; HR, hazard risk; LR, log rank; LUSC, lung squamous cell carcinoma.

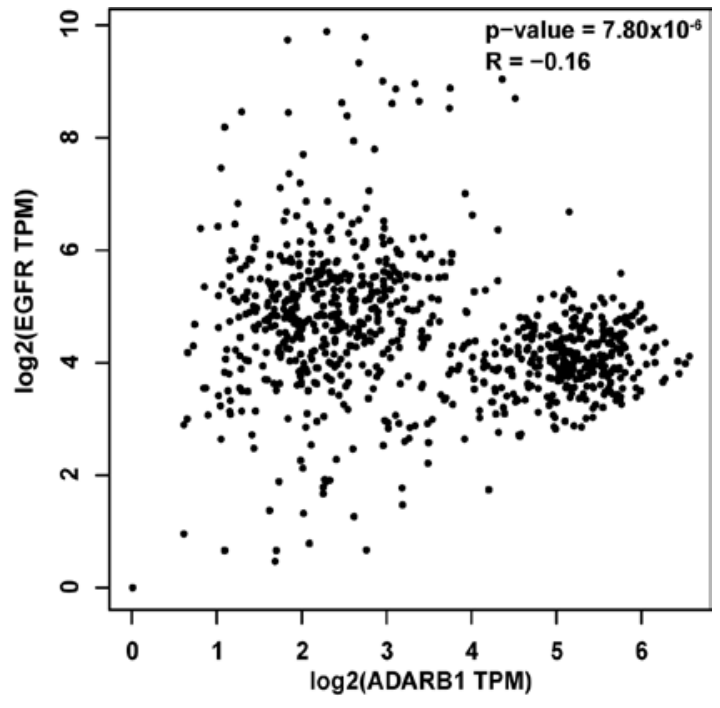

Figure 5. ADARB1 expression is negatively correlated with EGFR expression in LUSC. The Gene Expression Profiling Interactive Analysis database identified an association between the transcript levels of ADARB1 and EGFR in tissues derived from patients with LUSC. ADARB1, adenosine deaminase RNA specific B1; EGFR, epidermal growth factor receptor; LUSC, lung squamous cell carcinoma; TPM, transcripts per million.

and 'protein binding', respectively. Finally, KEGG pathway enrichment was analyzed using the DAVID database, and 'ubiquitin mediated proteolysis' and 'Wnt signaling pathway' were identified as significantly enriched pathways related to ADARB1 expression (Table SIV).

\section{Discussion}

The present study was conducted to evaluate the use of ADARB1 as a potential biomarker for patients with LUSC. To the best of our knowledge, the present study was the first to use bioinformatics methods to suggest that ADARB1 was downregulated in LUSC tissues and cell lines. Moreover, the expression of ADARB1 in LUSC was significantly different to that in LUAD. In addition, the global methylation levels of ADARB1 were upregulated in LUSC and a higher methylation value of $\operatorname{cg} 24063645$ was related to a shorter OS in patients with LUSC.

A-to-I RNA editing is a post-transcriptional modification mediated by the ADAR family, which is comprised of three members, ADAR1, ADARB1 and ADAR3 $(36,37)$. The expression levels of ADARB1 determine the RNA editing activity at a number of A-to-I sites, which are involved in several diseases, including amyotrophic lateral sclerosis, autism and Fragile-X Syndrome (38). Recently, several studies have indicated that ADARB1 participates in tumorigenesis as a cancer suppressor gene, which is consistent with the results of the present study. By analyzing the influence of A-to-I RNA modification on cancer progression, Fritzell et al (39) reported that ADARB1 was expressed at low levels in glioblastoma, indicating that ADARB1 downregulation could induce malignant phenotypes. Additionally, using next-generation sequencing transcriptomics, Chan et al (40) reported that ADARB1 contributed to inhibitory mechanisms in gastric cancer via its catalytic deaminase. However, Altadill et al (41) demonstrated that ADARB1 was upregulated in endometrial cancer, and the 
Table III. Association between the clinical characteristics and methylation site value of cg24063645 of adenosine deaminase RNA specific B1 in patients with lung squamous cell carcinoma.

\begin{tabular}{|c|c|c|c|}
\hline Variable & $\mathrm{n}$ & Mean \pm SD & P-value \\
\hline Sex & & & 0.717 \\
\hline Male & 215 & $0.983 \pm 0.042$ & \\
\hline Female & 73 & $0.985 \pm 0.021$ & \\
\hline KRAS gene analysis indicator & & & 0.760 \\
\hline Yes & 6 & $0.988 \pm 0.002$ & \\
\hline No & 197 & $0.983 \pm 0.038$ & \\
\hline EGFR mutation status & & & 0.682 \\
\hline Yes & 15 & $0.987 \pm 0.002$ & \\
\hline No & 174 & $0.983 \pm 0.040$ & \\
\hline Treatment outcome: First course & & & 0.718 \\
\hline Complete remission/response & 55 & $0.983 \pm 0.022$ & \\
\hline Stable disease & 2 & $0.989 \pm 0.001$ & \\
\hline Pathologic T & & & 0.562 \\
\hline $\mathrm{T} 1 / \mathrm{T} 1 \mathrm{a} / \mathrm{T} 1 \mathrm{~b}$ & 76 & $0.986 \pm 0.009$ & \\
\hline $\mathrm{T} 2 / \mathrm{T} 2 \mathrm{a} / \mathrm{T} 2 \mathrm{~b}$ & 166 & $0.980 \pm 0.049$ & \\
\hline $\mathrm{T} 3$ & 37 & $0.987 \pm 0.004$ & \\
\hline $\mathrm{T} 4$ & 9 & $0.988 \pm 0.002$ & \\
\hline Pathologic N & & & 0.117 \\
\hline No & 179 & $0.984 \pm 0.029$ & \\
\hline $\mathrm{N} 1$ & 79 & $0.985 \pm 0.014$ & \\
\hline $\mathrm{N} 2$ & 24 & $0.967 \pm 0.099$ & \\
\hline NX & 6 & $0.984 \pm 0.004$ & \\
\hline Pathologic M & & & 0.861 \\
\hline M0 & 233 & $0.984 \pm 0.035$ & \\
\hline M1/M1a & 3 & $0.988 \pm 0.003$ & \\
\hline MX & 50 & $0.986 \pm 0.004$ & \\
\hline Tumor stage & & & 0.919 \\
\hline I/IA/IB & 141 & $0.986 \pm 0.015$ & \\
\hline IIA/IIB & 96 & $0.986 \pm 0.013$ & \\
\hline IIIA/IIIB & 46 & $0.976 \pm 0.072$ & \\
\hline IV & 3 & $0.988 \pm 0.003$ & \\
\hline Ethnicity & & & 0.878 \\
\hline White & 224 & $0.984 \pm 0.028$ & \\
\hline Black/African American & 10 & $0.988 \pm 0.002$ & \\
\hline Asian & 7 & $0.988 \pm 0.003$ & \\
\hline Karnofsky score & & & 0.025 \\
\hline $0-70$ & 29 & $0.988 \pm 0.002$ & \\
\hline 80 & 14 & $0.987 \pm 0.002$ & \\
\hline 90 & 18 & $0.985 \pm 0.004$ & \\
\hline 100 & 9 & $0.987 \pm 0.002$ & \\
\hline Age & & & 0.341 \\
\hline$\leq 60$ years & 59 & $0.987 \pm 0.003$ & \\
\hline$>60$ years & 221 & $0.982 \pm 0.043$ & \\
\hline
\end{tabular}

Values in bold are significant. EGFR, epidermal growth factor receptor.

increased expression was positively associated with disease aggression, suggesting that ADARB1 functions as an oncogene in endometrial cancer. The inconsistency between the observations of these aforementioned studies and the present study may 

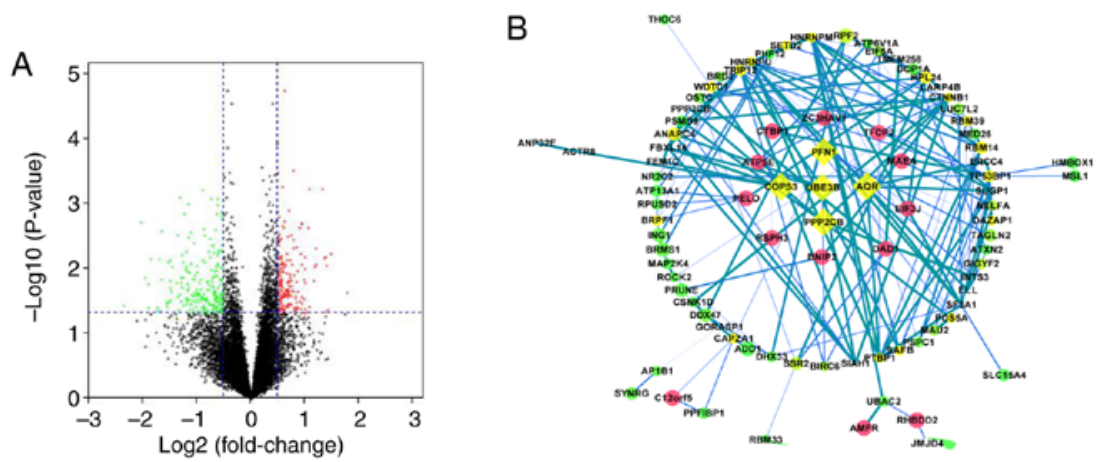

C Bar chart of biological process categories Bar chart of cellular component categories Bar chart of molecular function categories
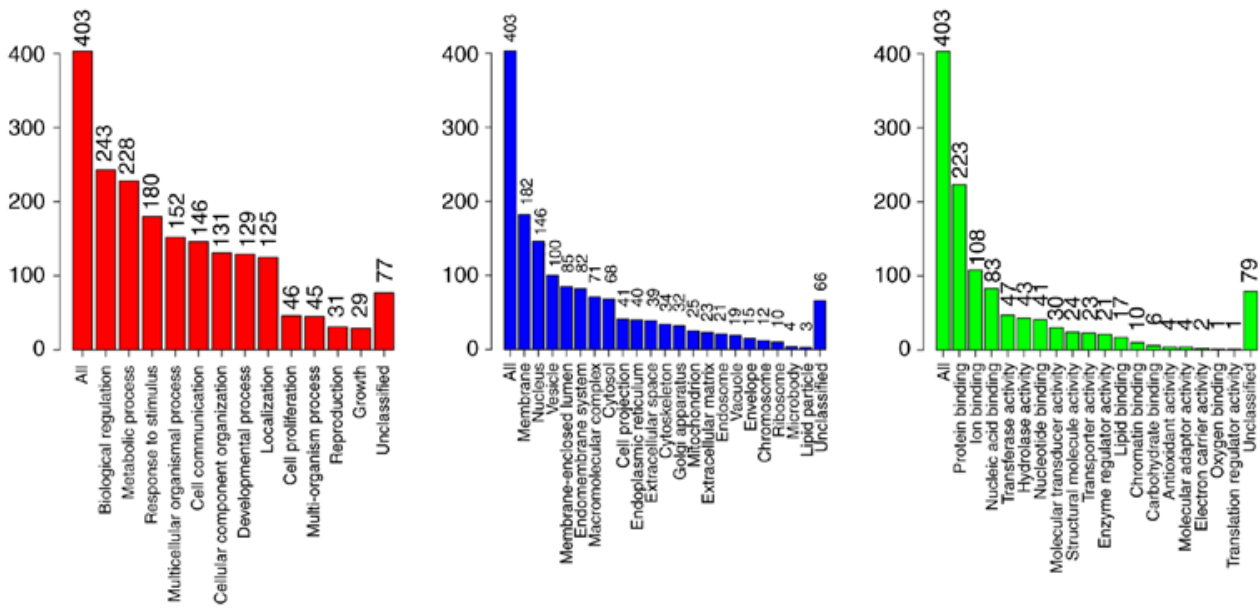

Figure 6. Functional enrichment analysis of ADARB1-associated co-DEGs in LUSC samples. (A) Volcano plot displayed the genes co-expressed with ADARB1 in LUSC (red represents upregulation, green represents downregulation and black is no change). (B) A protein-protein interaction network of ADARB1-associated co-DEGs was generated using the Search Tool for the Retrieval of Interacting Genes/Proteins database and Cytoscape software (square nodes represent hub genes, round nodes represent co-expression genes; the color represents the degree score; degree score $<0.5$ represent low values (colored yellow), degree score $\geq 0.5$ represent high values (other colors). (C) Gene Ontology analysis of ADARB1-associated co-DEGs. ADARB1, adenosine deaminase RNA specific B1; co-DEGs, co-differentially expressed genes; LUSC, lung squamous cell carcinoma.

be due to the diverse pathological states of different diseases; therefore, further investigation is required.

ADARB1 is a therapeutic target in a number of human diseases. Uchida et al (42) reported a significant increase in ADAR2-dependent A-to-I editing at the glutamate ionotropic receptor AMPA type subunit R/G, 5-hydroxytryptamine 2c receptor $D$ and COPI coat complex- $\alpha \mathrm{I} / \mathrm{V}$ sites, which suggested that ADARB1 was a regulator of neuropathic pain after peripheral nerve injury and could be used as a target for the treatment of neuropathic pain. In addition, Galeano et al (43) reported that ADARB1 could inhibit glioblastoma growth by the regulation of the cell division cycle 14B/S-phase kinase associated protein 2/p21/p27 axis; therefore, ADARB1 or its substrates represented suitable targets for the treatment of glioblastoma. In the present study, ADARB1 was significantly downregulated in LUSC and was associated with clinical characteristics of patients with LUSC. Furthermore, the hypermethylation of ADARB1 was related to the poor prognosis of patients with LUSC. Therefore, it was speculated that ADARB1 may represent a potential target for the treatment of LUSC; however, this requires further investigation.

LUSC and LUAD are the two main subtypes of NSCLC, and the accurate differentiation of LUSC and LUAD is important for therapy stratification (44). Although p40 is regarded as the gold standard for the differentiation of LUSC and
LUAD by the World Health Organization and the European Society for Medical Oncology (45-47), the identification of further biomarkers is required for distinguishing LUSC from LUAD. At present, numerous immune markers have been reported for the differentiation of LUSC and LUAD, including NK2 homeobox 1, napsin A, keratin 5 and p63. Kriegsmann et al and van Zyl et al $(48,49)$ reported that all four aforementioned markers were differentially expressed in LUSC and LUAD, and could therefore be used as biomarkers for the two cancer subtypes. By immunohistochemistry and western blotting, Wang et al (50) reported that the expression of the transcriptional co-factor tafazzin was associated with subtypes of LUSC and LUAD. Additionally, spermatogenesis associated serine rich 2, which is highly expressed in both LUSC and LUAD, displayed significantly higher expression in LUSC than LUAD, and thus, could be used as a marker to discriminate between the two subtypes $(51,52)$. Similarly, in the present study, ADARB1 expression was significantly higher in LUAD tissues and cell lines compared with in LUSC tissues. Therefore, the present study suggested that ADARB1 might be used to distinguish between LUSC and LUAD.

EGFR has been demonstrated to be a driving factor in various types of cancer. A previous study indicated that, following phosphorylation on a regulatory tyrosine, EGFR can be activated in a ligand-dependent or -independent manner to 
induce tumor progression (53). Additionally, EGFR has been reported to be upregulated in a number of different types of cancer and has been associated with cancer metastasis, drug resistance, poor prognosis and lower survival rates (54). Moreover, the combination of aberrantly expressed EGFR and transforming growth factor (TGF)- $\alpha$ secretion by NSCLC cells can form a TGF- $\alpha$-EGFR autocrine ring, which promotes tumor development $(55,56)$. EGFR can also activate the downstream PI3K/Akt/mTOR signaling pathway, leading to cell growth and proliferation during NSCLC (57). The present study identified a negative correlation between ADARB1 and EGFR expression in LUSC. Thus, it was hypothesized that ADARB1 could disturb the EGFR carcinogenic effect via metabolic processes and biological regulation in LUSC. This observation suggested that ADARB1 might act as an anti-oncogene by influencing EGFR expression; therefore, ADARB1 may be a useful molecular target for LUSC therapeutics.

In summary, the expression and role of ADARB1 in LUSC was investigated using a novel bioinformatics method. The results suggested that ADARB1 may be an anti-oncogene, and that it could be a promising biomarker in the tumorigenesis of LUSC and development of novel therapeutics for the disease. Therefore, understanding the function and mechanisms of ADARB1 in LUSC would provide a useful basis for the diagnosis and treatment of LUSC.

\section{Acknowledgements}

Not applicable.

\section{Funding}

The present study was supported by grants from the National Natural Science Foundation of China (grant nos. 81703036, 81803035 and 81572946), the Fundamental Research Funds for the Central Universities of Central South University (grant no. 2019zzts345), the China Postdoctoral Science Foundation (grant no. 2017M610510) and the Youth Fund of Xiangya Hospital (grant no. 2017Q17).

\section{Availability of data and materials}

The datasets used and/or analyzed during the current study are available from the corresponding author on reasonable request.

\section{Authors' contributions}

$Z X, Y Y$ and $Z G$ conceived and designed the study. XW, YY and ZX developed the methodology. XR, XW and WL acquired the data. ZX, XW, YY, XC and JW analyzed and interpreted the data. XW, XR and ZX wrote, reviewed and revised the manuscript.

\section{Ethics approval and consent to participate}

Not applicable.

\section{Patient consent for publication}

Not applicable.

\section{Competing interests}

The authors declare that they have no competing interests.

\section{References}

1. Yan Y, Xu Z, Qian L, Zeng S, Zhou Y, Chen X, Wei J and Gong Z: Identification of CAV1 and DCN as potential predictive biomarkers for lung adenocarcinoma. Am J Physiol Lung Cell Mol Physiol 316: L630-L643, 2019.

2. Zheng H, Zhan Y, Liu S, Lu J, Luo J, Feng J and Fan S: The roles of tumor-derived exosomes in non-small cell lung cancer and their clinical implications. J Exp Clin Cancer Res 37: 226, 2018.

3. Brahmer J, Reckamp KL, Baas P, Crinò L, Eberhardt WE, Poddubskaya E, Antonia S, Pluzanski A, Vokes EE, Holgado E, et al: Nivolumab versus docetaxel in advanced squamous-cell non-small-cell lung cancer. N Engl J Med 373: 123-135, 2015.

4. Herbst RS, Baas P, Kim DW, Felip E, Pérez-Gracia JL, Han JY, Molina J, Kim JH, Arvis CD, Ahn MJ, et al: Pembrolizumab versus docetaxel for previously treated, PD-L1-positive, advanced non-small-cell lung cancer (KEYNOTE-010): A randomised controlled trial. Lancet 387: 1540-1550, 2016.

5. Zugazagoitia J, Ponce S and Paz-Ares L: Necitumumab for first-line treatment of advanced, squamous, non-small-cell lung cancer: A relevant step forward? Transl Lung Cancer Res 5: 95-97, 2016.

6. Drilon A, Rekhtman N, Ladanyi M and Paik P: Squamous-cell carcinomas of the lung: Emerging biology, controversies and the promise of targeted therapy. Lancet Oncol 13: e418-e426, 2012.

7. Yan Y, Xu Z, Hu X, Qian L, Li Z, Zhou Y, Dai S, Zeng S and Gong Z: SNCA is a functionally low-expressed gene in lung adenocarcinoma. Genes 9: pii: E16, 2018.

8. Tan WL, Jain A, Takano A, Newell EW, Iyer NG, Lim WT, Tan EH, Zhai W, Hillmer AM, Tam WL and Tan DSW: Novel therapeutic targets on the horizon for lung cancer. Lancet Oncol 17: e347-e362, 2016.

9. Wang C, Zou J, Ma X, Wang E and Peng G: Mechanisms and implications of ADAR-mediated RNA editing in cancer. Cancer Lett 411: 27-34, 2017.

10. Peng L, Zhang H, Su Y, Shen Q, Du C, Xie H, Li H, Yan J, Shen Z, Jiang W, et al: Lipopolysaccharide enhances ADAR2 which drives Hirschsprung's disease by impairing miR-142-3p biogenesis. J Cell Mol Med 22: 4045-4055, 2018.

11. Chen YB, Liao XY, Zhang JB, Wang F, Qin HD, Zhang L, Shugart YY, Zeng YX and Jia WH: ADAR2 functions as a tumor suppressor via editing IGFBP7 in esophageal squamous cell carcinoma. Int J Oncol 50: 622-630, 2017.

12. Chen YT, Chang IY, Liu H, Ma CP, Kuo YP, Shih CT, Shih YH, Kang L and Tan BC: Tumor-associated intronic editing of HNRPLL generates a novel splicing variant linked to cell proliferation. J Biol Chem 293: 10158-10171, 2018.

13. Kuner R, Muley T, Meister M, Ruschhaupt M, Buness A, Xu EC, Schnabel P, Warth A, Poustka A, Sültmann H and Hoffmann H: Global gene expression analysis reveals specific patterns of cell junctions in non-small cell lung cancer subtypes. Lung Cancer 63: 32-38, 2009.

14. Barrett T and Edgar R: Reannotation of array probes at NCBI's GEO database. Nat Methods 5: 117, 2008.

15. Tang $Z$, Li C, Zhang K, Yang $M$ and Hu X: GE-mini: A mobile APP for large-scale gene expression visualization. Bioinformatics 33: 941-943, 2017.

16. Barretina J,Caponigro G,Stransky N, Venkatesan K, Margolin AA, Kim S, Wilson CJ, Lehár J, Kryukov GV, Sonkin D, et al: The cancer cell line encyclopedia enables predictive modelling of anticancer drug sensitivity. Nature 483: 603-607, 2012.

17. Rhodes DR, Yu J, Shanker K, Deshpande N, Varambally R, Ghosh D, Barrette T, Pandey A and Chinnaiyan AM: ONCOMINE: A cancer microarray database and integrated data-mining platform. Neoplasia 6: 1-6, 2004.

18. Chandrashekar DS, Bashel B, Balasubramanya $\mathrm{SAH}$, Creighton CJ, Ponce-Rodriguez I, Chakravarthi BVSK and Varambally S: UALCAN: A portal for facilitating tumor subgroup gene expression and survival analyses. Neoplasia 19: 649-658, 2017.

19. Tang Z, Li C, Kang B, Gao G, Li C and Zhang Z: GEPIA: A web server for cancer and normal gene expression profiling and interactive analyses. Nucleic Acids Res 45: W98-W102, 2017. 
20. Diez-Villanueva A, Mallona I and Peinado MA: Wanderer, an interactive viewer to explore DNA methylation and gene expression data in human cancer. Epigenetics Chromatin 8: 22, 2015.

21. Xiong Y, Wei Y, Gu Y, Zhang S, Lyu J, Zhang B, Chen C, Zhu J, Wang Y, Liu $\mathrm{H}$ and Zhang Y: DiseaseMeth version 2.0: A major expansion and update of the human disease methylation database. Nucleic Acids Res 45: D888-D895, 2017.

22. Modhukur V, Iljasenko T, Metsalu T, Lokk K, Laisk-Podar T and Vilo J: MethSurv: A web tool to perform multivariable survival analysis using DNA methylation data. Epigenomics 10: 277-288, 2018

23. Cancer Genome Atlas Research Network: Comprehensive genomic characterization of squamous cell lung cancers Nature 489: 519-525, 2012.

24. Gao J, Aksoy BA, Dogrusoz U, Dresdner G, Gross B, Sumer SO, Sun Y, Jacobsen A, Sinha R, Larsson E, et al: Integrative analysis of complex cancer genomics and clinical profiles using the cBioPortal. Sci Signal 6: pl1, 2013.

25. Szklarczyk D, Morris JH, Cook H, Kuhn M, Wyder S, Simonovic M, Santos A, Doncheva NT, Roth A, Bork P, et al: The STRING database in 2017: Quality-controlled protein-protein association networks, made broadly accessible. Nucleic Acids Res 45: D362-D368, 2017.

26. Reimand J, Isserlin R, Voisin V, Kucera M, Tannus-Lopes C, Rostamianfar A, Wadi L, Meyer M, Wong J, Xu C, et al Pathway enrichment analysis and visualization of omics data using g:Profiler, GSEA, Cytoscape and EnrichmentMap. Nat Protoc 14: 482-517, 2019.

27. Kanehisa M, Furumichi M, Tanabe M, Sato Y and Morishima K: KEGG: New perspectives on genomes, pathways, diseases and drugs. Nucleic Acids Res 45: D353-D361, 2017.

28. Wang J, Vasaikar S, Shi Z, Greer M and Zhang B: WebGestalt 2017: A more comprehensive, powerful, flexible and interactive gene set enrichment analysis toolkit. Nucleic Acids Res 45: W130-W137, 2017.

29. Huang da W, Sherman BT and Lempicki RA: Systematic and integrative analysis of large gene lists using DAVID bioinformatics resources. Nat Protoc 4: 44-57, 2009.

30. Yang L, Liu L, Xu Z, Liao W, Feng D, Dong X, Xu S, Xiao L, Lu J, Luo X, et al: EBV-LMP1 targeted DNAzyme enhances radiosensitivity by inhibiting tumor angiogenesis via the JNKs/HIF-1 pathway in nasopharyngeal carcinoma. Oncotarget 6: 5804-5817, 2015.

31. Wang X, Xu Z, Ren X, Chen X, Wei J, Lin W, Li Z, Ou C, Gong Z and Yan Y: Function of low ADARB1 expression in lung adenocarcinoma. PLoS One 14: e0222298, 2019.

32. Zhou S, Yan Y, Chen X, Wang X, Zeng S, Qian L, Wei J, Yang X, Zhou Y, Gong Z and Xu Z: Roles of highly expressed PAICS in lung adenocarcinoma. Gene 692: 1-8, 2019.

33. Xiao D, Lu C, Zhu W, He Q, Li Y, Fu C, Zhou J, Liu S and Tao Y: Comparison of small biopsy specimens and surgical specimens for the detection of EGFR mutations and EML4-ALK in non-small-cell lung cancer. Oncotarget 7: 59049-59057, 2016.

34. Gan Z, Zhao L, Yang L, Huang P, Zhao F, Li W and Liu Y: RNA editing by ADAR2 is metabolically regulated in pancreatic islets and beta-cells. J Biol Chem 281: 33386-33394, 2006

35. Li Z, Tian Y, Tian N, Zhao X, Du C, Han L and Zhang H: Aberrant alternative splicing pattern of ADAR2 downregulates adenosine-to-inosine editing in glioma. Oncol Rep 33 2845-2852, 2015

36. Merkle T, Merz S, Reautschnig P, Blaha A, Li Q, Vogel P, Wettengel J, Li JB and Stafforst T: Precise RNA editing by recruiting endogenous ADARs with antisense oligonucleotides. Nat Biotechnol 37: 133-138, 2019.

37. Kuang L, Lv G, Wang B, Li L, Dai Y and Li Y: Overexpression of adenosine deaminase acting on RNA 1 in chordoma tissues is associated with chordoma pathogenesis by reducing miR $125 \mathrm{a}$ and miR10a expression. Mol Med Rep 12: 93-98, 2015.

38. Hosaka T, Yamashita T, Teramoto S, Hirose N, Tamaoka A and Kwak S: ADAR2-dependent A-to-I RNA editing in the extracellular linear and circular RNAs. Neurosci Res 147: 48-57, 2019.

39. Fritzell K, Xu LD, Lagergren J and Öhman M: ADARs and editing: The role of A-to-I RNA modification in cancer progression. Semin Cell Dev Biology 79: 123-130, 2018

40. Chan TH, Qamra A, Tan KT, Guo J, Yang H, Qi L, Lin JS, $\mathrm{Ng} \mathrm{VH}$, Song Y, Hong H, et al: ADAR-Mediated RNA editing predicts progression and prognosis of gastric cancer. Gastroenterology 151: 637-650.e10, 2016.
41. Altadill T, Dowdy TM, Gill K, Reques A, Menon SS, Moiola CP, Lopez-Gil C, Coll E, Matias-Guiu X, Cabrera S, et al: Metabolomic and Lipidomic profiling identifies the role of the RNA editing pathway in endometrial carcinogenesis. Sci Rep 7: 8803, 2017.

42. Uchida H, Matsumura S, Okada S, Suzuki T, Minami T and Ito S: RNA editing enzyme ADAR2 is a mediator of neuropathic pain after peripheral nerve injury. FASEB J 31: 1847-1855, 2017.

43. Galeano F, Rossetti C, Tomaselli S, Cifaldi L, Lezzerini M, Pezzullo M, Boldrini R, Massimi L, Di Rocco CM, Locatelli F and Gallo A: ADAR2-editing activity inhibits glioblastoma growth through the modulation of the CDC14B/Skp2/p21/p27 axis. Oncogene 32: 998-1009, 2013.

44. Chen M, Liu X, Du J, Wang XJ and Xia L: Differentiated regulation of immune-response related genes between LUAD and LUSC subtypes of lung cancers. Oncotarget 8: 133-144, 2017.

45. Planchard D, Popat S, Kerr K, Novello S, Smit EF, Faivre-Finn C, Mok TS, Reck M, Van Schil PE, Hellmann MD and Peters S: Metastatic non-small cell lung cancer: ESMO Clinical Practice Guidelines for diagnosis, treatment and follow-up. Ann Oncol 30: 863-870, 2019.

46. Bishop JA, Teruya-Feldstein J, Westra WH, Pelosi G, Travis WD and Rekhtman N: p40 ( $\Delta$ Np63) is superior to p63 for the diagnosis of pulmonary squamous cell carcinoma. Mod Pathol 25: 405-415, 2012.

47. Travis WD, Brambilla E, Burke AP, Marx A and Nicholson AG: Introduction to The 2015 World Health Organization classification of tumors of the lung, pleura, thymus, and heart. J Thorac Oncol 10: 1240-1242, 2015 .

48. Kriegsmann K, Cremer M, Zgorzelski C, Harms A, Muley T, Winter H, Kazdal D, Warth A and Kriegsmann M: Agreement of CK5/6, p40, and p63 immunoreactivity in non-small cell lung cancer. Pathology 51: 240-245, 2019.

49. van Zyl A, Schubert PT and Koegelenberg CFN: The utility of TTF-1, napsin A, CK5 and p63 staining in the sub-classification of non-small cell carcinoma of the lung. Cytopathology 30: 586-591, 2019.

50. Wang Y, Han Y, Guo Z, Yang Y and Ren T: Nuclear TAZ activity distinctly associates with subtypes of non-small cell lung cancer. Biochem Biophys Res Commun 509: 828-832, 2019.

51. Hou J,Aerts J,den Hamer B, van Ijcken W, den Bakker M, Riegman P, van der Leest C, van der Spek P, Foekens JA, Hoogsteden HC, et al: Gene expression-based classification of non-small cell lung carcinomas and survival prediction. PLoS One 5: e10312, 2010.

52. Takamochi K, Ohmiya H, Itoh M, Mogushi K, Saito T, Hara K, Mitani K, Kogo Y, Yamanaka Y, Kawai J, et al: Novel biomarkers that assist in accurate discrimination of squamous cell carcinoma from adenocarcinoma of the lung. BMC Cancer 16: 760, 2016.

53. Lipsick J: A history of cancer research: Tyrosine kinases. Cold Spring Harb Perspect Biol 11: pii: a035592, 2019.

54. Martinez P, Peters S, Stammers T and Soria JC: Immunotherapy for the First-line treatment of patients with metastatic non-small cell lung cancer. Clin Cancer Res 25: 2691-2698, 2019.

55. Liu Y, Jiang H, Zhou H, Ying X, Wang Z, Yang Y, Xu W, He X and $\mathrm{Li} \mathrm{Y}$ : Lentivirus-mediated silencing of HOTAIR lncRNA restores gefitinib sensitivity by activating Bax/Caspase-3 and suppressing TGF- $\alpha /$ EGFR signaling in lung adenocarcinoma. Oncol Lett 15: 2829-2838, 2018.

56. Romero-Ventosa EY, Blanco-Prieto S, Gonzalez-Pineiro AL, Rodriguez-Berrocal FJ, Pineiro-Corrales G and Paez de la Cadena M: Pretreatment levels of the serum biomarkers CEA, CYFRA 21-1, SCC and the soluble EGFR and its ligands EGF, TGF-alpha, HB-EGF in the prediction of outcome in erlotinib treated non-small-cell lung cancer patients. Springerplus 4: 171,2015

57. Gao J, Qiu X, Xi G, Liu H, Zhang F, Lv T and Song Y: Downregulation of GSDMD attenuates tumor proliferation via the intrinsic mitochondrial apoptotic pathway and inhibition of EGFR/Akt signaling and predicts a good prognosis in non-small cell lung cancer. Oncol Rep 40: 1971-1984, 2018

This work is licensed under a Creative Commons Attribution-NonCommercial-NoDerivatives 4.0 International (CC BY-NC-ND 4.0) License. 